\title{
Influência do comprimento das seções no ajuste de funções de afilamento
}

Este trabalho teve como objetivo verificar a ocorrência de diferenças nas estimativas de diâmetro, altura e volume em diferentes níveis de amostragem por meio de diferentes comprimentos de seções, considerando ou não a influência da base com o uso de funções de afilamento. No processo de cubagem, foram medidos 70 fustes do híbrido Eucayptus urophylla x Eucalyptus grandis, com 8 anos de idade. Para efeito do estudo, considerou-se dez níveis de amostragem separados por comprimentos de seções de 0,5 m, 1,0 m, 1,5 m, 2,0 m, 2,5 m, 3,0 m, 3,5 m, 4,0 m, 4,5 m e 5,0. Foi ajustado o modelo de afilamento de Demaerschalk em cada um dos níveis amostrados, considerando ou não a influência da base para obtenção das estimativas dos parâmetros utilizados na estimação das variáveis. A escolha do nível de amostragem mais preciso, considerando ou não a influência da base, foi feita pela análise gráfica dos resíduos, do coeficiente de determinação ( $R^{\wedge} 2$ ), do erro padrão da estimativa (Syx (\%)), do viés (V), da média das diferenças (MD) e do desvio padrão das diferenças (DPD). Os resultados mostraram que o nível de amostragem de $0,5 \mathrm{~m}$ foi mais preciso em estimar as variáveis analisadas, principalmente no cálculo do volume. Ficou evidenciado também que as estimativas considerando a influência da base tiveram resultados mais precisos em relação ao grupo que não considerou esta influência. Dessa forma, concluiu-se que seções menores com mais seções medidas na base tem estimativas mais acuradas de diâmetro, altura e volume.

Palavras-chave: Níveis de Amostragem; Cubagem; Influência da Base.

\section{Influence of sections length in fit taper functions}

\begin{abstract}
This work had as objective to verify the occurrence of differences in the estimates of diameter, height and volume at different levels of sampling using different sections lengths, considering or not the influence of the base with the use of taper functions. In the process of cubage, 70 stems of the Eucalyptus urophylla $x$ Eucayptus grandis hybrid were measured, with 8 years-old. For this study, ten sampling levels were considered, separated into sections of lengths of $0,5 \mathrm{~m}, 1,0 \mathrm{~m}$, $1,5 \mathrm{~m}, 2,0 \mathrm{~m}, 2,5 \mathrm{~m}, 3,0 \mathrm{~m}, 3,5 \mathrm{~m}, 4,0 \mathrm{~m}, 4,5 \mathrm{~m}$ and 5,0 m. It was adjusted the Demaerschalk taper model in each sampled levels, considering or not the influence of the basis for obtaining parameters estimates used in the variables estimation. Choosing the precision level of sampling, considering or not the influence of the base, was performed by graphic analysis of the waste, the determination coefficient $\left(\mathrm{R}^{\wedge} 2\right)$, estimated standard error (Syx $\left.(\%)\right)$, bias $(\mathrm{V})$, average of the differences (MD) and standard deviation of the differences (DPD). The results showed that the sampling level of $0,5 \mathrm{~m}$ was more accurate in estimating the analyzed variables, especially in the volume calculation. It was proven that the estimates considering the influence of the base had results indicating greater accuracy than the group that did not consider this influence. Thus, it was concluded that smaller sections with more sections measured at the base have more accurate estimates of diameter, height and volume.
\end{abstract}

Keywords: Levels of Sampling; Cubage; Influence of Base.

Topic: Ciências Florestais

Reviewed anonymously in the process of blind peer.
Received: 06/05/2020 Approved: 04/06/2020
Rômulo Môra (iD)

Universidade Federal de Mato Grosso, Brasil http://lattes.cnpq.br/3456015172126546 http://orcid.org/0000-0002-2234-6753 romulomef@yahoo.com.br

Gilson Fernandes da Silva (iD)

Universidade Federal do Espírito Santo, Brasil http://lattes.cnpq.br/8643263800313625 http://orcid.org/0000-0001-7853-6284

fernandes5012@gmail.com

Fabricio Gomes Gonçalves (iD

Universidade Federal do Espírito Santo, Brasil http://lattes.cnpq.br/0616694853822879 http://orcid.org/0000-0003-2010-9508

fabriciogomes@ibest.com.br

\author{
José Franklim Chichorro (D) \\ Universidade Federal do Espírito Santo, Brasil \\ http://lattes.cnpq.br/0368239095423222 \\ http://orcid.org/0000-0003-3592-3238 \\ jfranklim@cca.ufes.br \\ Carlos Pedro Boechat Soares (iD) \\ Universidade Federal de Viçosa, Brasil \\ http://lattes.cnpq.br/0959425632265455 \\ http://orcid.org/0000-0001-6475-3376 \\ csoares@ufv.br \\ Leandro Tose Martins (iD) \\ Universidade Federal do Espírito Santo, Brasil \\ http://lattes.cnpq.br/6734041235674996 \\ http://orcid.org/0000-0003-2160-798X \\ Itosemartins@hotmail.com
}

Referencing this:

MÔRA, R.; SILVA, G. F.; GONÇALVES, F. G.; CHICHORRO, J. F.; SOARES, C. P. B.; MARTINS, L. T.. Influência do comprimento das seções no ajuste de funções de afilamento. Revista Ibero Americana de Ciências Ambientais, v.11, n.4, p.36-48, 2020. DOI:

http://doi.org/10.6008/CBPC2179-6858.2020.004.0003 


\section{INTRODUÇÃO}

A diversificação na oferta de produtos é um parâmetro essencial na mensuração do desenvolvimento florestal. Segundo Angelo et al. (1995), para propiciar diferentes usos ao tronco de uma árvore, é necessário conhecer sua forma, a qual determinará o método de processamento na definição das seções de cubagem. Quantificar os estoques volumétricos tem sido o principal objetivo do mapeamento de florestas. Campos et al. (2009) sugerem a utilização de procedimentos de inventário baseado em amostra, em que o volume da árvore é estimado por meio de equações volumétricas e outros procedimentos, tais como modelos de razão ou modelos de afilamento.

Os métodos mais comuns de ajustes volumétricos são obtidos com dados de cubagem das árvores amostra (SOARES et al., 2006). Como a árvore não tem a forma de um cilindro perfeito, conhecer seu perfil torna-se necessário na estimativa do volume entre cada seção, para quantificar os produtos e assim determinar o lucro esperado pela produção.

Há várias opiniões de autores a respeito das fórmulas mais precisas em relação às seções de dimensionamento nas árvores (SOARES et al., 2010). As mais utilizadas são as de Newton, Huber e Smalian. Goulding (1979) e Husch et al. (2003) afirmam que Newton é a fórmula mais precisa, pois leva em conta as diferentes formas que o tronco da árvore pode assumir: cilindro, neiloide, paraboloide e cone. Finger (1992) destaca que as fórmulas de Smalian e Huber são precisas apenas quando o tronco se assemelha a um paraboloide.

De acordo com Soares et al. (2010), há muitas fórmulas diferentes para determinar o volume da árvore e de galhos e também diferentes resultados decorrentes da sua utilização. No entanto, pouca atenção tem sido dada à influência do comprimento da seção em volume. Dessa forma, objetivou-se neste trabalho verificar a influência do comprimento das seções nas estimativas das variáveis diâmetro, altura e volume em diferentes níveis de amostragem considerando ou não a influência da base.

\section{MATERIAIS E MÉTODOS}

\section{Local de estudo}

O estudo foi realizado no Instituto Federal de Educação, Ciência e Tecnologia de Minas Gerais (IFMG) - Campus São João Evangelista, localizado no município de São João Evangelista, no estado de Minas Gerais. A altitude média ao nível do mar no Campus é de 452 metros, temperatura média de 22ํㅡㄴ Celsius com máxima anual de 26,1ำ Celsius e com mínima de 15 Celsius. O índice médio pluviométrico anual é de $1.081 \mathrm{~mm}$. A classificação do clima segundo Köppen é Cwa com inverno seco e verão chuvoso.

\section{Banco de dados}

As medições foram realizadas num talhão de 3,48 hectares plantado com o híbrido Eucalyptus urophylla $\times$ Eucalyptus grandis proveniente de propagação clonal estabelecido num espaçamento de $3 \times 2$ metros, com 8 anos de idade. 
O processo de cubagem consistiu em medir a circunferência $(c)$ em seções de 0,5 em 0,5 m até atingir a altura (h) do diâmetro mínimo da ponta da árvore de 4,0 centímetros. Esse seccionamento foi realizado em 70 árvores com dap que variaram de $4,5 \mathrm{~cm}$ até $32,6 \mathrm{~cm}$ e alturas totais que variaram de 8,68 até 31,79 metros. Para cada árvore, mediu-se também a circunferência a 1,30 m do solo (cap) e a altura total ( $h t)$. 0 volume total (v) foi obtido se somando os volumes parciais de todas as seções até o diâmetro mínimo de 4 $\mathrm{cm}$, utilizando a metodologia de cubagem rigorosa absoluta de Smalian.

\section{Metodologia de Amostragem}

As estimativas das variáveis diâmetro e altura, utilizando o banco de dados contendo $d, h$, dap, e $h t$, em estudo neste trabalho foram feitas utilizando o modelo de Demaerschalk com o uso dos coeficientes ajustados das expressões de diâmetro e altura do modelo, obtidas a partir do rearranjo das variáveis do modelo original de Demaerschalk, e o volume foi estimado a partir da integração da expressão diâmetro, nos diferentes níveis de amostragem, representados pelos diferentes comprimentos de seções, do fuste da árvore. Este modelo foi escolhido dentre outros ajustados para este conjunto de dados, pois verificada as estatísticas $R^{2}$, Syx (\%) e análise gráfica de resíduos, este apresentou melhores resultados quando comparado a Baldwin, Kozak e Ormerod (MÔRA et al., 2014). O modelo de Demaerschalk foi caracterizado com as seguintes expressões relativas às variáveis $d, h$ e $v$ descritas a seguir:

$$
\begin{aligned}
& \hat{d}=10^{\widehat{\beta}_{0}} \operatorname{dap}^{\widehat{\beta}_{1}} h t^{\widehat{\beta}_{2}}(h t-h)^{\widehat{\beta}_{3}} \\
& \hat{h}=h t-\left[\frac{d}{10^{\widehat{\beta}_{0} D A P^{\widehat{\beta}_{1}} h t^{\widehat{\beta}_{2}}}}\right]^{\left(\frac{1}{\beta_{3}}\right)} \\
& \hat{V}=\frac{\pi}{40000} 10^{2 \widehat{\beta}_{0}} \operatorname{dap}^{2 \widehat{\beta}_{1}} h t^{2 \widehat{\beta}_{2}}\left[\frac{\left(h t-h_{1}\right)^{2 \widehat{\beta}_{3}+1}-\left(h t-h_{2}\right)^{2 \widehat{\beta}_{3}+1}}{2 \widehat{\beta}_{3}+1}\right]
\end{aligned}
$$

Em que: $d=$ diâmetro com casca na altura $h$; dap = diâmetro com casca medido na altura de $1,30 \mathrm{~m} ; h=$ distância do solo até o ponto onde o diâmetro d é considerado; $h t=$ altura total; $h_{1}=$ altura correspondente a primeira seção $(0,0$ m); $h_{2}=$ altura correspondente a seção quando o diâmetro mínimo atinge 4 centímetros. $\beta_{i}=$ parâmetros do modelo ajustado (i=0, 1, 2,3); e $V$ = volume obtido para a seção desejada.

Para efeito deste estudo, foram considerados dez níveis de amostragem que correspondem a diferentes comprimentos de seções medidas nas árvores. Esses comprimentos de seções foram medidos nos fustes, começando da base da árvore (0,0 m), em intervalos de 0,5 m, 1,0 m, 1,5 m, 2,0 m, 2,5 m, 3,0 m, 3,5 m, 4,0 m, 4,5 m e 5,0 m, até o fuste atingir um diâmetro mínimo de 4,0 centímetros.

Admitindo que a maior porção do volume esteja contida na base do fuste e que também há uma maior variação da forma nessa porção, os dados para ajuste das funções de afilamento foram divididos em dois grupos. No sentido de se verificar a influência da porção basal na qualidade do ajuste das funções de afilamento, um dos grupos considerou os dados medidos na base no ajuste das funções de afilamento (G1) e o outro grupo não levou em consideração a medição de um número maior de seções na base (G2). Por exemplo, para a cubagem feita no fuste de uma árvore com 29,0 m de altura para o nível de amostragem em intervalos de 4,0 m, para o $\mathrm{G} 1$ foram medidas as seções em 0,0 m, 0,5 m, 1,0 m, 1,5 m, 2,0 m, 4,0 m, 8,0 m, $12,0 \mathrm{~m}, 16,0 \mathrm{~m}, 20,0 \mathrm{~m}, 24,0 \mathrm{~m}, 28,0 \mathrm{~m}$ e 29,0m, quando o fuste atingiu um diâmetro mínimo de $4 \mathrm{~cm}$. Já para esse mesmo fuste no $\mathrm{G} 2$, as cubagens foram realizadas nas seções de $0,0 \mathrm{~m}, 4,0 \mathrm{~m}, 8,0 \mathrm{~m}, 12,0 \mathrm{~m}, 16,0$ 
m, 20,0 m, 24,0 m, 28,0 m e 29,0m. Pelo exemplo apresentado, percebe-se que no G1 a influência da base é obtida acrescentando-se os níveis de amostragem correspondentes às seções de 0,5 m, 1,0 m, 1,5 m e 2,0 m.

Tendo em vista o exposto, foram ajustadas equações para cada nível de amostragem para as três variáveis em estudo $(d, h$ e $v)$. Para evitar equívocos na comparação entre os resultados de diâmetro e altura dado o número diferente de observações para um mesmo nível de amostragem, os valores das seções correspondentes à base que não estavam presentes no conjunto de dados do $\mathrm{G} 2$ no ajuste da função foram introduzidos no banco de dados de cada nível de amostragem e calculadas suas estimativas, obtendo assim o mesmo número de dados para análise. Para o volume, isso não foi necessário, já que a estimação dessa variável leva em consideração apenas $h_{1}$ correspondente a seção de $0,0 m$ e $h_{2}$ correspondente à altura no diâmetro mínimo de 4 centímetros.

Os valores das estimativas dos parâmetros para todos os níveis de amostragem foram testados pelo teste ' $t$ ' de modo a verificar a significância dos parâmetros estimados para as equações relativas às variáveis em estudo ( $d, h$ e $v)$ a $5 \%$ de probabilidade. Os ajustes dos dez níveis de amostragem foram realizados no software STATISTICA da empresa Statsoft.

\section{Avaliação das estimativas}

Para as variáveis diâmetro e altura, os valores de todas as seções de todos os níveis de amostragem foram estimados por meio dos coeficientes obtidos das equações de diâmetro e altura após rearranjo das variáveis no modelo de Demaerschalk ajustadas para os dois grupos em todos os níveis de amostragem. Já a variável volume, a precisão foi verificada pelo cálculo do volume total considerando então como $h_{1}$ a base da árvore e $h_{2}$ a altura atingida quando o diâmetro mínimo atingido foi de 4,0 cm para todos os níveis de amostragem analisados com e sem influência da base.

A precisão das estimativas das variáveis foi feita com base no coeficiente de determinação $\left(R^{2}\right)$ e do erro padrão da estimativa em porcentagem $\left(S_{y x}(\%)\right)$. O coeficiente de determinação $\left(R^{2}\right)$ foi calculado da seguinte forma proposto por Kvalseth (1985):

$$
R^{2}=1-\frac{\sum_{i=1}^{n}\left(Y_{i}-\widehat{Y}\right)^{2}}{\sum_{i=1}^{n}\left(Y_{i}-\bar{Y}\right)^{2}} 100
$$

em que: $Y_{i}=$ i-ésimo valor observado para a variável dependente; $\widehat{Y}_{l}=$ i-ésimo valor estimado para a variável dependente Y; e $\bar{Y}=$ média dos valores observados para a variável dependente $\mathrm{Y}$;

Para todos os níveis estudados foram feitos testes de acordo com a metodologia utilizada por Ferreira (1999) e Souza et al. (2008). Primeiramente, foram feitas as análises gráficas dos resíduos. Os valores residuais utilizados na construção dos gráficos foram expressos por:

$$
\operatorname{Erro}(\%)=\frac{(Y-\hat{Y})}{Y} 100
$$

em que: $\hat{Y}=$ valores estimados pela equação e $Y=$ valores observados.

Para complementar a análise gráfica de resíduos, foram realizados testes complementares (Tabela 1), por meio das seguintes estatísticas: viés $(V)$, média das diferenças absolutas $(M D)$ e desvio padrão das diferenças $(D P D)$. A partir da análise das estatísticas $V, M D$ e $D P D$, procedeu-se a ordenação dos níveis de 
amostragem segundo o maior ou menor grau de precisão, sendo atribuídos pesos de 1 a 10 de acordo com os resultados das estatísticas obtidas para cada nível de amostragem em cada grupo. Foi considerado o nível de amostragem mais acurado aquele que resultou em menor somatório para cada situação analisada, levando em consideração que a estimativa mais precisa no nível de amostragem analisado teve atribuído o menor valor para cada estatística.

Tabela 1: Critérios para avaliação do ajuste e validação dos dados.

\begin{tabular}{|c|c|c|}
\hline Viés $(V)$ & Média das diferenças absolutas (MD) & Desvio padrão das diferenças absolutas (DPD) \\
\hline$V=\frac{\sum_{i=1}^{n} Y_{i}-\sum_{i=1}^{n} \hat{Y}_{i}}{n}$ & $M D=\frac{\sum_{i=1}^{n}\left|Y_{i}-\hat{Y}_{i}\right|}{n}$ & $D P D=\frac{\sum_{i=1}^{n} d_{i}^{2}-\frac{\left(\sum_{i=1}^{n} d_{i}\right)^{2}}{n}}{n-1}$ \\
\hline
\end{tabular}

Em que: $Y_{i}=$ volume calculado $\widehat{Y}_{l}$ valor estimado; $n=$ número de observações; e $p$ = número de parâmetros de cada modelo; e $d_{i}=Y_{i}-\widehat{Y}_{l}$

Para a determinação do nível de amostragem mais preciso, a análise isolada das estatísticas $V, M D$, $D P D$ não é conveniente pelo fato delas se complementarem na análise. Nesse sentido, a escolha do melhor método de amostragem foi feita pela classificação proposta pelo menor somatório das estatísticas em cada processo de estimação da variável conjuntamente com $R^{2}$, Syx(\%) e análise gráfica de resíduos.

\section{RESULTADOS}

\section{Para a variável diâmetro}

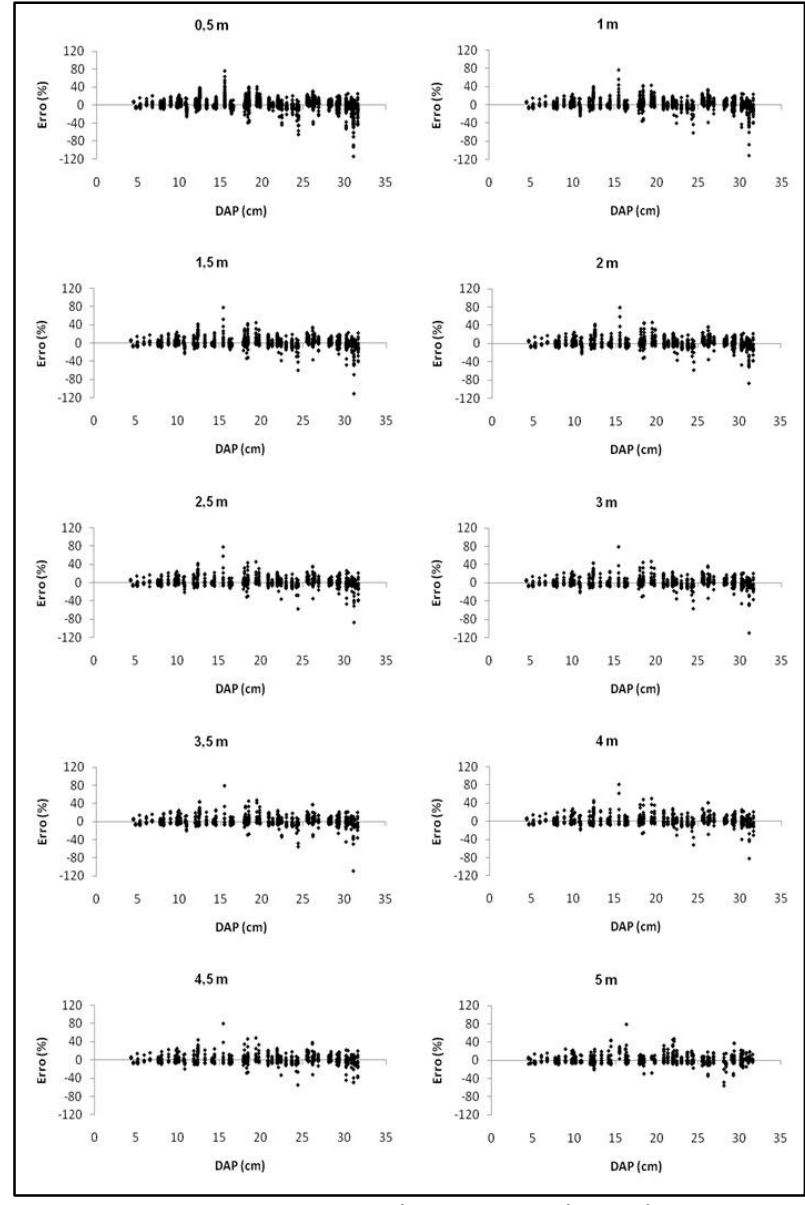

Figura 1: Distribuição dos resíduos da variável diâmetro, em porcentagem, em função do dap, para os dez níveis de amostragem analisados com a influência da base (G1).

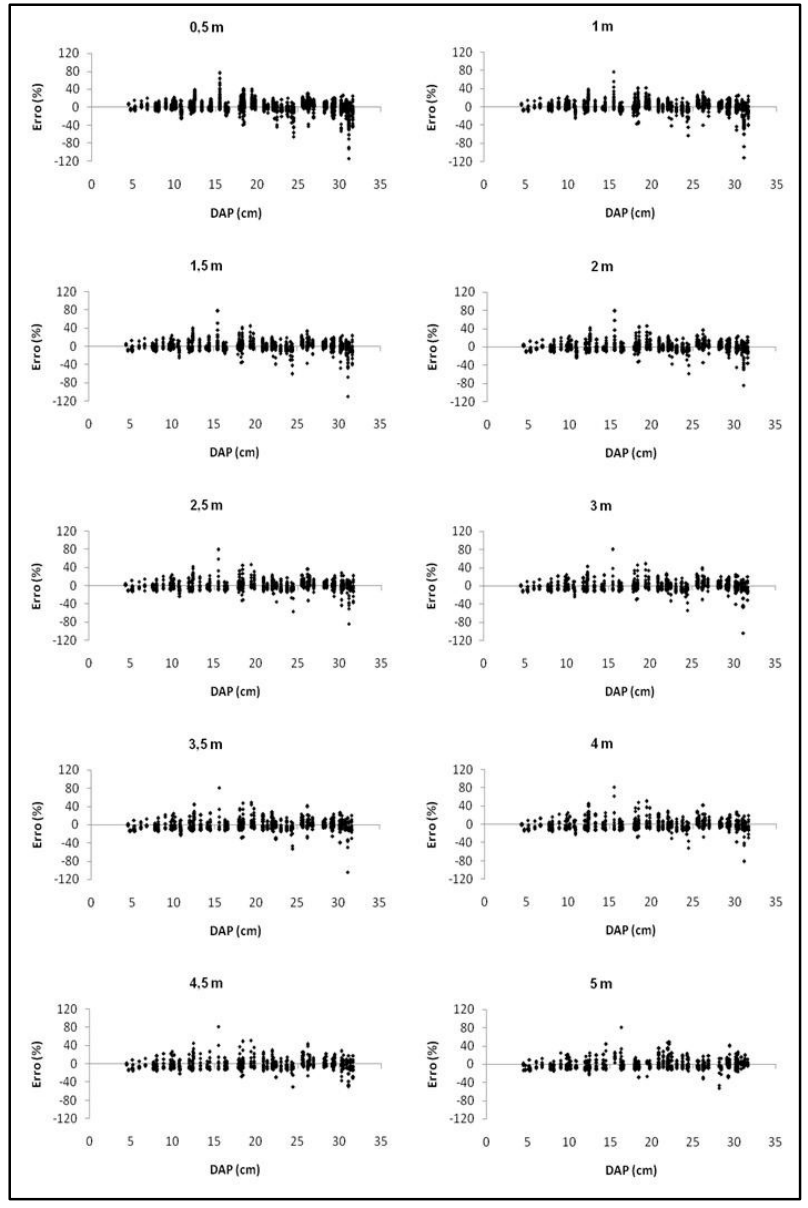

Figura 2: Distribuição dos resíduos da variável diâmetro, em porcentagem, em função do dap, para os dez níveis de amostragem analisados sem a influência da base (G2). 
Tabela 2: Estatísticas $R^{2}$, Syx $(\%)$, viés $(V)$, média das diferenças absolutas $(M D)$, desvio padrão das diferenças $(D P D)$ e a classificação para as estimativas de diâmetro para os ajustes com influência (G1) e sem influência da base (G2).

\begin{tabular}{|c|c|c|c|c|c|c|}
\hline \multicolumn{7}{|c|}{ Ajuste com influência da base } \\
\hline $\begin{array}{l}\text { Comprimento de } \\
\text { seções }\end{array}$ & $R^{2}$ & Syx (\%) & $V$ & $M D$ & $D P D$ & Classificação \\
\hline $0,5 \mathrm{~m}$ & 96,02 & 8,97 & $0,0329(1)$ & $0,8490(1)$ & $1,2508(1)$ & 1 \\
\hline $1,0 \mathrm{~m}$ & 96,16 & 9,29 & $0,0413(2)$ & $0,9014(2)$ & $1,3244(2)$ & 2 \\
\hline $1,5 \mathrm{~m}$ & 96,37 & 9,34 & $0,0439(3)$ & $0,9245(3)$ & $1,3586(3)$ & 3 \\
\hline $2,0 \mathrm{~m}$ & 96,48 & 9,44 & $0,0499(7)$ & $0,9488(5)$ & $1,3861(4)$ & 4 \\
\hline $2,5 \mathrm{~m}$ & 96,66 & 9,26 & $0,0462(4)$ & $0,9386(4)$ & $1,3911(5)$ & 5 \\
\hline $3,0 \mathrm{~m}$ & 96,72 & 9,30 & $0,0481(5)$ & $0,9556(6)$ & $1,4042(6)$ & 6 \\
\hline $3,5 \mathrm{~m}$ & 96,78 & 9,35 & $0,0505(8)$ & $0,9730(7)$ & $1,4186(7)$ & 7 \\
\hline $4,0 \mathrm{~m}$ & 96,84 & 9,32 & $0,1108(10)$ & $0,9846(9)$ & $1,4193(8)$ & 9 \\
\hline $4,5 \mathrm{~m}$ & 96,89 & 9,23 & $0,0484(6)$ & $0,9785(8)$ & $1,4265(9)$ & 8 \\
\hline $5,0 \mathrm{~m}$ & 96,86 & 9,40 & $0,0550(9)$ & $0,9857(10)$ & $1,4514(10)$ & 10 \\
\hline \multicolumn{7}{|c|}{ Ajuste sem influência da base } \\
\hline $\begin{array}{l}\text { Comprimento de } \\
\text { seções }\end{array}$ & $R^{2}$ & Syx (\%) & V & $M D$ & $D P D$ & Classificação \\
\hline $0,5 \mathrm{~m}$ & 96,02 & 8,97 & $0,0329(3)$ & $0,8490(1)$ & $1,2508(1)$ & 1 \\
\hline $1,0 \mathrm{~m}$ & 96,14 & 9,31 & $0,0510(4)$ & $0,9002(2)$ & $1,3263(2)$ & 2 \\
\hline $1,5 \mathrm{~m}$ & 96,34 & 9,37 & $0,0201(2)$ & $0,9395(3)$ & $1,3642(3)$ & 3 \\
\hline $2,0 \mathrm{~m}$ & 96,44 & 9,50 & $-0,0103(1)$ & $0,9797(4)$ & $1,3946(4)$ & 4 \\
\hline $2,5 \mathrm{~m}$ & 96,55 & 9,40 & $-0,1114(5)$ & $1,0146(5)$ & $1,4085(5)$ & 5 \\
\hline $3,0 \mathrm{~m}$ & 96,55 & 9,53 & $-0,1747(6)$ & $1,0606(6)$ & $1,4303(6)$ & 6 \\
\hline $3,5 \mathrm{~m}$ & 96,52 & 9,72 & $-0,2446(7)$ & $1,1191(7)$ & $1,4554(7)$ & 7 \\
\hline $4,0 \mathrm{~m}$ & 96,50 & 9,81 & $-0,3032(8)$ & $1,1461(8)$ & $1,4678(8)$ & 8 \\
\hline $4,5 \mathrm{~m}$ & 96,39 & 9,95 & $-0,3820(9)$ & $1,1927(9)$ & $1,4888(9)$ & 9 \\
\hline $5,0 \mathrm{~m}$ & 96,27 & 10,25 & $-0,4257(10)$ & $1,2309(10)$ & $1,5256(10)$ & 10 \\
\hline
\end{tabular}

Os valores dos erros relativos em porcentagem das estimativas da variável diâmetro em todos os níveis de amostragem para os grupos G1 e G2, estão, respectivamente, na Figura 1 e 2 . Os resultados de $R^{2}$, Syx(\%), $V, M D$ e $D P D$ para todos os níveis de amostragem nos dois grupos de dados estão na Tabela 2.

\section{Para a variável altura}

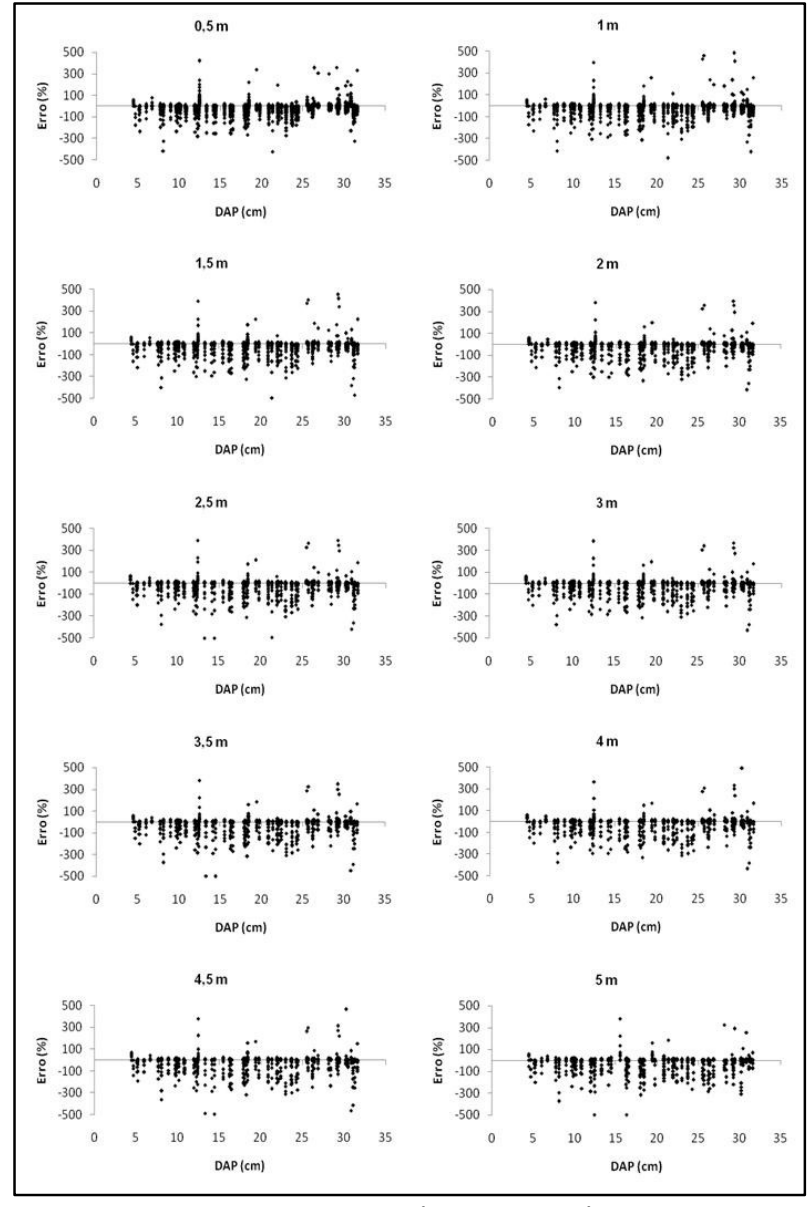

Figura 3: Distribuição dos resíduos da variável altura, em

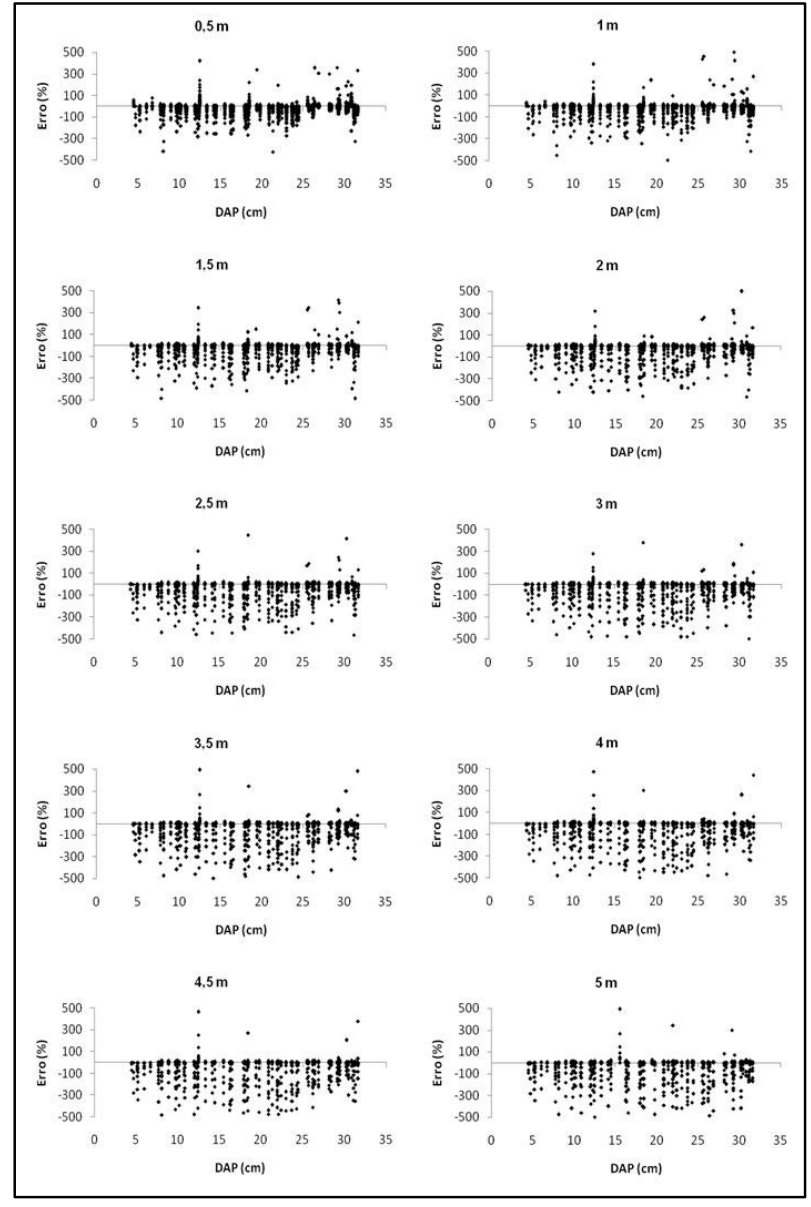

Figura 4: Distribuição dos resíduos da variável altura, em 
porcentagem, em função do dap, para os dez níveis de amostragem analisados com a influência da base (G1). porcentagem, em função do dap, para os dez níveis de amostragem analisados sem a influência da base (G2).

A análise gráfica de resíduos das estimativas de altura para todos os níveis de amostragem nos grupos 1 e 2 estão representados, respectivamente, nas Figuras 3 e 4. Na Tabela 3, estão os resultados das estatísticas de precisão analisadas para os grupos com e sem influência da base.

Tabela 3: Estatísticas $R^{2}$, Syx(\%), viés $(V)$, média das diferenças absolutas $(M D)$, desvio padrão das diferenças $(D P D)$ e a classificação para as estimativas de altura para os ajustes com influência (G1) e sem influência da base (G2).

\begin{tabular}{|c|c|c|c|c|c|c|}
\hline \multicolumn{7}{|c|}{ Ajuste com influência da base } \\
\hline $\begin{array}{l}\text { Comprimento de } \\
\text { seções }\end{array}$ & $R^{2}$ & Syx (\%) & $V$ & $M D$ & $D P D$ & Classificação \\
\hline $0,5 \mathrm{~m}$ & 94,61 & 15,07 & $0,0577(1)$ & $1,2735(1)$ & $1,7282(1)$ & 1 \\
\hline $1,0 \mathrm{~m}$ & 94,67 & 17,11 & $0,0741(2)$ & $1,3558(2)$ & $1,8180(2)$ & 2 \\
\hline $1,5 \mathrm{~m}$ & 94,73 & 18,99 & $0,0810(3)$ & $1,3968(3)$ & $1,8673(3)$ & 3 \\
\hline $2,0 \mathrm{~m}$ & 94,81 & 20,07 & 0,0908 (4) & $1,4320(4)$ & $1,8968(4)$ & 4 \\
\hline $2,5 \mathrm{~m}$ & 94,64 & 22,37 & $0,0939(5)$ & $1,4494(5)$ & $1,9228(5)$ & 5 \\
\hline $3,0 \mathrm{~m}$ & 94,64 & 23,39 & $0,0965(6)$ & $1,4724(6)$ & $1,9385(6)$ & 6 \\
\hline $3,5 \mathrm{~m}$ & 94,72 & 24,07 & $0,1021(7)$ & $1,4955(7)$ & $1,9500(7)$ & 7 \\
\hline $4,0 \mathrm{~m}$ & 94,70 & 24,88 & $0,1492(10)$ & $1,5076(9)$ & $1,9525(8)$ & 9 \\
\hline $4,5 \mathrm{~m}$ & 94,63 & 25,99 & $0,1051(8)$ & $1,4970(8)$ & $1,9586(9)$ & 8 \\
\hline $5,0 \mathrm{~m}$ & 94,53 & 26,82 & $0,1127(9)$ & $1,5273(10)$ & $1,9848(10)$ & 10 \\
\hline \multicolumn{7}{|c|}{ Ajuste sem influência da base } \\
\hline $\begin{array}{l}\text { Comprimento de } \\
\text { seções }\end{array}$ & $R^{2}$ & Syx (\%) & $V$ & $M D$ & $D P D$ & Classificação \\
\hline $0,5 \mathrm{~m}$ & 94,61 & 15,07 & $0,0577(2)$ & $1,2735(1)$ & $1,7282(1)$ & 1 \\
\hline $1,0 \mathrm{~m}$ & 94,65 & 17,13 & $0,0071(1)$ & $1,3661(2)$ & $1,8216(2)$ & 2 \\
\hline $1,5 \mathrm{~m}$ & 94,64 & 19,15 & $-0,1197(3)$ & $1,4454(3)$ & $1,8814(3)$ & 3 \\
\hline $2,0 \mathrm{~m}$ & 94,63 & 20,41 & $-0,2045(4)$ & $1,5101(4)$ & $1,9210(4)$ & 4 \\
\hline $2,5 \mathrm{~m}$ & 94,14 & 23,39 & $-0,4182(5)$ & $1,6044(5)$ & $1,9695(5)$ & 5 \\
\hline $3,0 \mathrm{~m}$ & 93,94 & 24,88 & $-0,5202(6)$ & $1,6617(6)$ & $1,9984(6)$ & 6 \\
\hline $3,5 \mathrm{~m}$ & 93,79 & 26,10 & $-0,6219(7)$ & $1,7256(7)$ & $2,0237(7)$ & 7 \\
\hline $4,0 \mathrm{~m}$ & 93,56 & 27,42 & $-0,7034(8)$ & $1,7627(8)$ & $2,0404(8)$ & 8 \\
\hline $4,5 \mathrm{~m}$ & 93,20 & 29,24 & $-0,8040(9)$ & $1,8055(9)$ & $2,0548(9)$ & 9 \\
\hline $5,0 \mathrm{~m}$ & 92,89 & 30,59 & $-0,8724(10)$ & $1,8623(10)$ & $2,0925(10)$ & 10 \\
\hline
\end{tabular}

\section{Para a variável volume}

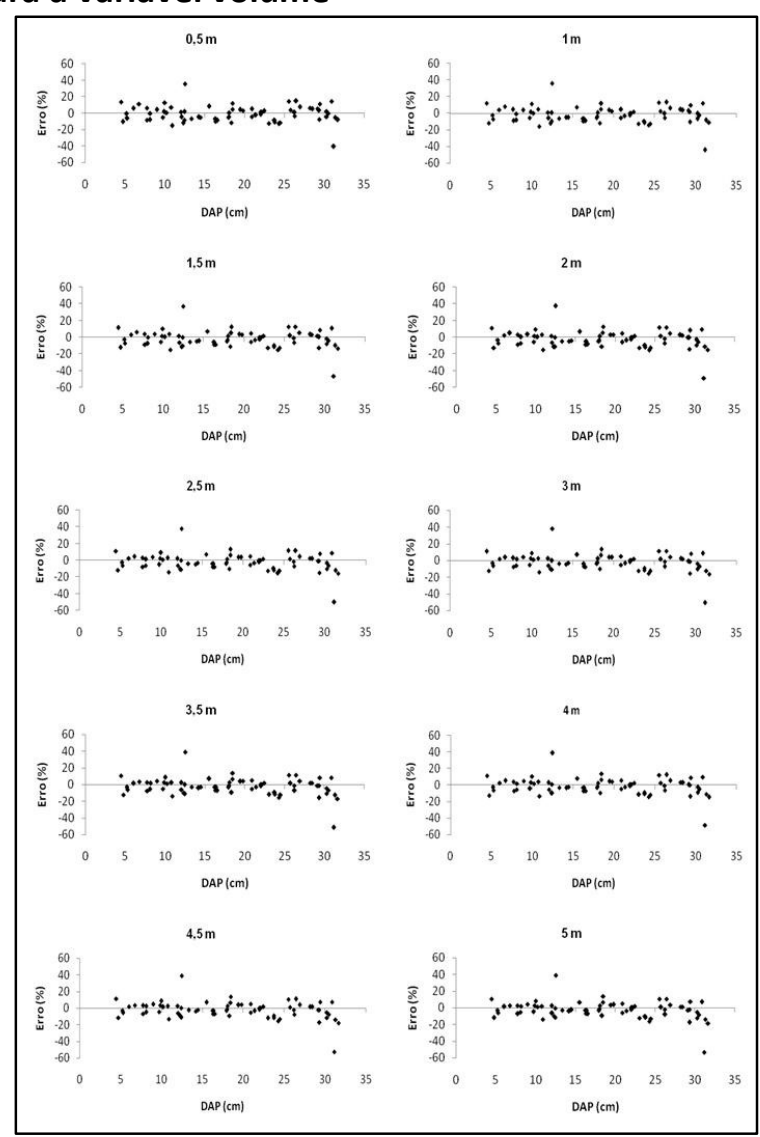

Figura 5: Distribuição dos resíduos da variável volume, em

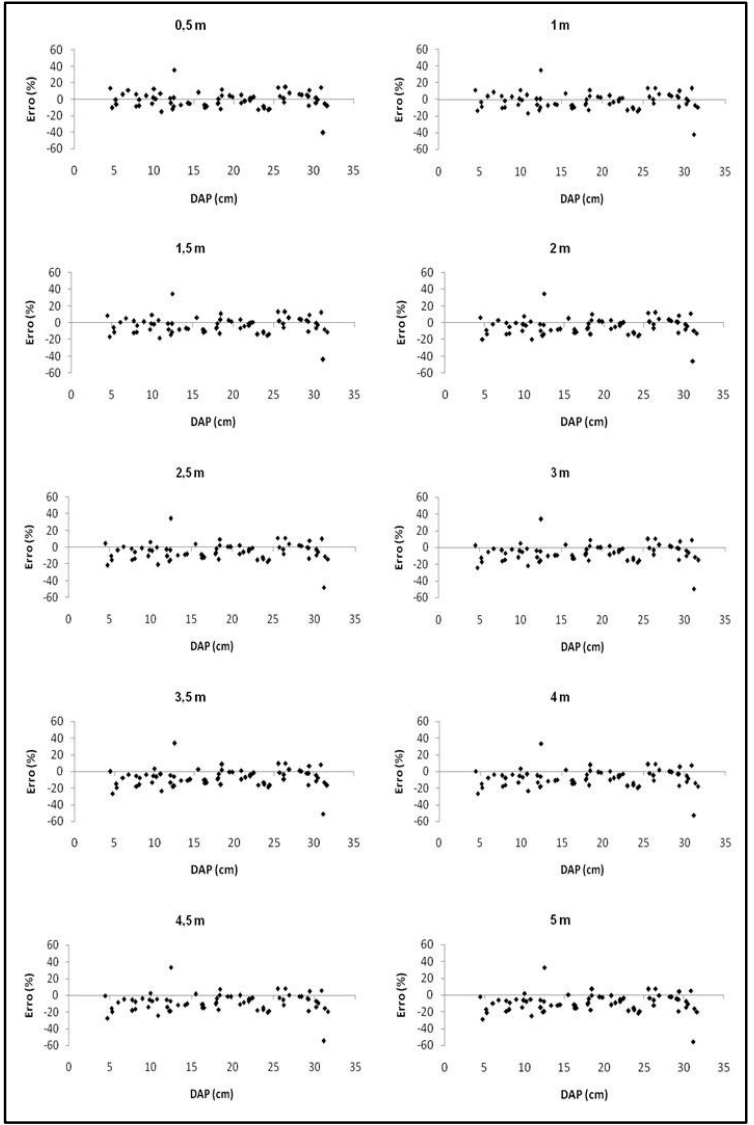

Figura 6: Distribuição dos resíduos da variável volume, em 
porcentagem, em função do dap, para os dez níveis de amostragem analisados com a influência da base (G1). porcentagem, em função do dap, para os dez níveis de amostragem analisados sem a influência da base (G2)

As Figuras 5 e 6 representam, respectivamente, a análise gráfica de resíduos da variável volume estimado com a influência da base (G1) e sem a influência da base (G2). Na Tabela 4, estão os resultados das estatísticas de precisão analisadas para os grupos com e sem influência da base.

Tabela 4: Estatísticas $R^{2}$, Syx $(\%)$, viés $(V)$, média das diferenças absolutas $(M D)$, desvio padrão das diferenças $(D P D)$ e a classificação para as estimativas de volume para os ajustes com influência (G1) e sem influência da base (G2).

\begin{tabular}{|c|c|c|c|c|c|c|}
\hline \multicolumn{7}{|c|}{ Ajuste com influência da base } \\
\hline $\begin{array}{l}\text { Comprimento de } \\
\text { Seções }\end{array}$ & $R^{2}$ & Syx (\%) & $V$ & $M D$ & $D P D$ & Classificação \\
\hline $0,5 \mathrm{~m}$ & 97,23 & 12,89 & $-0,0001(1)$ & $0,0275(2)$ & $0,0472(1)$ & 1 \\
\hline $1,0 \mathrm{~m}$ & 97,14 & 13,11 & $-0,0048(2)$ & $0,0273(1)$ & $0,0477(2)$ & 2 \\
\hline $1,5 \mathrm{~m}$ & 96,98 & 13,45 & $-0,0071(4)$ & $0,0276(3)$ & $0,0487(3)$ & 3 \\
\hline $2,0 \mathrm{~m}$ & 96,82 & 13,82 & $-0,0092(5)$ & $0,0279(4)$ & $0,0497(4)$ & 5 \\
\hline $2,5 \mathrm{~m}$ & 96,70 & 14,07 & $-0,0094(7)$ & $0,0282(5)$ & $0,0506(6)$ & 6 \\
\hline $3,0 \mathrm{~m}$ & 96,66 & 14,17 & $-0,0093(6)$ & $0,0283(6)$ & $0,0509(7)$ & 7 \\
\hline $3,5 \mathrm{~m}$ & 96,55 & 14,40 & $-0,0102(8)$ & $0,0286(7)$ & $0,0516(8)$ & 8 \\
\hline $4,0 \mathrm{~m}$ & 96,85 & 13,75 & $-0,0065(3)$ & $0,0276(3)$ & $0,0498(5)$ & 4 \\
\hline $4,5 \mathrm{~m}$ & 96,39 & 14,72 & $-0,0113(9)$ & $0,0290(8)$ & $0,0526(9)$ & 9 \\
\hline $5,0 \mathrm{~m}$ & 96,33 & 14,83 & $-0,0119(10)$ & $0,0291(9)$ & $0,0529(10)$ & 10 \\
\hline \multicolumn{7}{|c|}{ Ajuste sem influência da base } \\
\hline $\begin{array}{l}\text { Comprimento de } \\
\text { Seções }\end{array}$ & $R^{2}$ & Syx (\%) & V & $M D$ & $D P D$ & Classificação \\
\hline $0,5 \mathrm{~m}$ & 97,23 & 12,89 & $-0,0001(1)$ & $0,0275(1)$ & $0,0472(1)$ & 1 \\
\hline $1,0 \mathrm{~m}$ & 97,20 & 12,96 & $-0,0034(2)$ & $0,0275(1)$ & $0,0473(2)$ & 2 \\
\hline $1,5 \mathrm{~m}$ & 97,12 & 13,15 & $-0,0069(3)$ & $0,0279(2)$ & $0,0476(3)$ & 3 \\
\hline $2,0 \mathrm{~m}$ & 96,97 & 13,49 & $-0,0105(4)$ & $0,0286(3)$ & $0,0481(4)$ & 4 \\
\hline $2,5 \mathrm{~m}$ & 96,73 & 14,02 & $-0,0146(5)$ & $0,0298(4)$ & $0,0490(5)$ & 5 \\
\hline $3,0 \mathrm{~m}$ & 96,56 & 14,37 & $-0,0170(6)$ & $0,0308(5)$ & $0,0496(6)$ & 6 \\
\hline $3,5 \mathrm{~m}$ & 96,22 & 15,07 & $-0,0208(7)$ & $0,0326(6)$ & $0,0508(7)$ & 7 \\
\hline $4,0 \mathrm{~m}$ & 95,92 & 15,65 & $-0,0238(8)$ & $0,0343(7)$ & $0,0517(8)$ & 8 \\
\hline $4,5 \mathrm{~m}$ & 95,49 & 16,44 & $-0,0270(9)$ & $0,0362(8)$ & $0,0533(9)$ & 9 \\
\hline $5,0 \mathrm{~m}$ & 95,22 & 16,95 & $-0,0294(10)$ & $0,0378(9)$ & $0,0541(10)$ & 10 \\
\hline
\end{tabular}

\section{DISCUSSÃO}

\section{Para a variável diâmetro}

Na Figura 1, pode-se verificar uma semelhança entre as análises gráficas dos dez níveis de amostragem na estimação do variável diâmetro, mesmo com o número de dados diferentes devido ao número diferentes de seções medidas entre os níveis estudados. Pode-se ainda notar que, em todos os níveis de amostragem, o modelo tem uma leve tendência para superestimar diâmetros quando o dap $>30 \mathrm{~cm}$. $\mathrm{Na}$ Figura 2, pode-se notar, assim como na Figura 1, uma semelhança entre os erros relativos nos níveis de amostragem considerados. Observa-se também uma tendência em superestimar os diâmetros para dap > 30 $\mathrm{cm}$, assim como nos modelos com influência da base.

Comparando as duas Figuras, devido ao número de dados ser diferente em cada nível de amostragem, os gráficos dos erros para o diâmetro estimado pela expressão de diâmetro apresentam semelhança para tamanhos de seções iguais. Pode-se também observar pelos gráficos que não se pode perceber de maneira significativa que a base influenciou nas estimativas das variáveis. Desse modo, a escolha do nível de amostragem mais acurado na estimação dessa variável, deve ser feita utilizando a análise gráfica e as estatísticas da Tabela 2.

Na Tabela 2 os valores do $R^{2}$ foram altos e próximos nos diferentes níveis de amostragem. No G1, esses valores tiveram uma variação de $96,02 \%$ para o nível de $0,5 \mathrm{~m}$ a 96,89\% m para o nível de 4,5 m. No 
G2, essa variação foi um pouco menor de $96,02 \%$ para o nível de $0,5 \mathrm{~m}$ a 96,55\% para o nível de 2,5 m. Os resultados da estatística Syx (\%), para ambos os casos, foram precisos em todos os níveis de amostragem (não superando os 11\%). Porém, pode-se notar que os valores da estatística Syx (\%) no G2 tiveram, um crescimento superior, de acordo com a diminuição do número de seções amostradas em relação ao G1, que apresentou erros semelhantes entre os ajustes.

Pode-se notar que os resultados da estatística $R^{2}$ em comparação com Syx(\%), MD e DPD ficam contraditórios, pois a expectativa é de que com o aumento do $R^{2}$, haja uma diminuição do Syx(\%). Observase na Tabela 2 que muitas vezes a estatística $R^{2}$ não segue a tendência das estatísticas $\operatorname{Syx}(\%), M D$ e $D P D$ que tendem a piorar com a diminuição do número de seções amostradas, ficando mais evidenciado quando não se considera a influência da base. Isso pode ser explicado pelo fato do modelo de afilamento de Demaerschalk ser não linear e como mencionado por Regazzi (2003), para alguns modelos não lineares os valores de $R^{2}$ encontrados na literatura são, em sua maioria, próximos a 100\%, mesmo havendo enorme variação entre os valores observados e preditos. Segundo Regazzi (2010), um engano comumente mantido é a crença de que o $R^{2}$, a "proporção da variação explicada", é usado para decidir se um modelo de regressão não-linear resulta num bom ajuste aos dados e isso só ocorre quando se tem um modelo linear com o termo constante é que o $R^{2}$ representa a variação explicada pelo modelo. Ratkowsky (1990) afirmou que mesmo havendo enorme discrepância entre os valores observados e estimados, os modelos não-lineares podem, às vezes, apresentar valores de $R^{2}$ extremamente elevados $\left(R^{2}=99 \%\right)$.

Já as estatísticas $V, M D$ e $D P D$ mostram resultados com maior precisão e menos tendenciosos quando se considerou a influência da base no ajuste. Pela estatística viés, pode-se verificar que no caso de G1, os valores dessa estatística foram relativamente não tendenciosos, excluindo o caso do nível $4 \mathrm{~m}$, onde essa estatística teve valor de 0,1108. Para o G2, observa-se que os valores tendem a aumentar com a diminuição do número de seções medidas, mostrando para os níveis abaixo de $2 \mathrm{~m}$ uma tendência em subestimar a variável, enquanto desse nível de amostragem para os superiores a tendência da estimativa fica superestimada.

Para a estatística $M D$, assim como para a estatística viés, obteve-se resultados mais precisos no grupo G1, nos níveis de amostragem com seções menores, tendo valores relativamente baixos não superando a unidade. Resultado contrário ocorre em G2, onde as estimativas perdem a precisão, à medida que diminui o número de seções medidas no fuste. Comparando os ajustes, observa-se que o $\mathrm{G} 1$ teve resultados mais precisos e menos tendenciosos que o G2, verificando-se assim a influência positiva do maior número de seções medidas na base na estimativa da variável.

\section{Para a variável altura}

Na Figura 3, os resultados dos gráficos de resíduos para os níveis de amostragem no G1 são semelhantes. Estes apresentam resultados de superestimações em todas as classes de diâmetro para todos os níveis de amostragem. Isso aconteceu pelo fato desse grupo ter considerado a influência da base e como o modelo não tem precisão para estimar seções menores, aumentam assim os valores dos erros de 
superestimação, prejudicando as estatísticas.

$\mathrm{Na}$ Figura 4, pode-se observar em todas as estimativas nos níveis de amostragem uma tendência em superestimar a altura em todas as classes de diâmetro. Pode-se verificar que, à medida que os níveis de amostragem aumentam e há menor quantidade de seções medidas, as estimativas da variável altura superestimam com maior intensidade quando comparadas ao verdadeiro valor e como os modelos de afilamento não são precisos para estimar alturas em seções menores, os gráficos ficam com valores de erros relativos maiores, justificando o maior número de superestimações ocorridas.

Confrontando os resultados das duas Figuras, para o G1 e G2, nota-se que os erros relativos nos resultados sob a influência da base são menores do que o grupo sem a influência da base. Porém, isso não garante que a estimativas sejam melhores no G1 sobre o G2. Devem-se levar em consideração na definição do melhor nível de amostragem as análises de resíduos conjuntamente com as estatísticas $V, M D$ e $D P D$.

Na tabela 3 os valores de $R^{2}$ não tiveram resultados tão altos quanto para as outras variáveis em estudo, com valores entre 92 e 94\%, considerando ambos os grupos de dados em todos os níveis de amostragem. Já os valores de Syx(\%), assim como ocorreu para a variável diâmetro, foram melhores no grupo com influência da base, mesmo com valores altos (superior a 15\%). Os resultados dos valores do erro de amostragem do G2 tiveram um aumento superior em relação ao G1 à medida que diminuíam o número de seções medidas no fuste iniciando com um erro de $15,07 \%$ no nível de amostragem de 0,5 m chegando a 30,59\% no nível de amostragem de $5 \mathrm{~m}$. Esses valores indicam que o menor número de seções com comprimento de seções menores aumentam os valores dos erros, já que as equações de afilamento não são precisas para estimar a variável altura como ocorre com o diâmetro e volume. Os valores de $R^{2}$, assim como ocorreu para a variável diâmetro, tiveram resultados equivocados com as outras estatísticas.

Em relação aos resultados encontrados na estatística Syx(\%) para a variavel altura em comparação ao diâmetro, nota-se que para altura os valores dos erros aumentaram à medida que diminuíam o número de seções medidas. Para a variável diâmetro os erros foram próximos em relação aos diferentes níveis de amostragem. No G2, pode-se ainda verificar que Syx(\%) teve seu valor dobrado quando se compara o nível de $0,5 \mathrm{~m}$ com o nível de $5 \mathrm{~m}$. Outro fato importante a se considerar é que mesmo com a dificuldade de estimar alturas menores, os resultados dessa estatística foram melhores quando se considerou a influência da base no ajuste, demonstrando assim que mais medições na base contribui para melhores estimativas no cálculo das alturas ao longo do fuste.

Assim como aconteceu para a variável diâmetro, na estatística viés, a tendência dos erros tende a aumentar com a dimuição do número de seções medidas no tronco para ambos os grupos de dados, mostrando tendência em estimar essa variável. Na estatística média das diferenças, os valores de $M D$ indicaram menor precisão (superiores a 1,2735), indicando assim que os ajustes não tiveram precisão nas estimativas obtidas, como pode ser visto na análise gráfica de resíduos, havendo uma tendência em superestimar a altura em todos os níveis de amostragem para ambos os casos. Ao observar os grupos, e desconsiderar algumas variações nas estatísticas, pode-se notar que para essa variável, o grupo com influência da base teve resultados mais precisos, mesmo com o maior número de seções com comprimentos 
de menor magnitude.

\section{Para a variável volume}

Pelos resultados apresentados na Figura 5, o nível de amostragem de 0,5 apresentou erros relativos menores que os outros níveis de amostragem estudados. Os outros níveis mostram uma tendência em superestimar os volumes, à medida que se aumenta o tamanho das seções de medição no fuste. Observa-se também que com a diminuição do número de seções medidas no fuste, os volumes estimados tendem a superestimar os volumes encontrados pela fórmula de Smalian. Soares et al. (2010), analisando as estimativas de volume total de Eucalyptus grandis para seções de 1, 2 e $3 \mathrm{~m}$ de comprimento, concluíram que o aumento do tamanho das seções do fuste leva a superestimações da parte basal e consequentemente, no volume total.

Pelos resultados apresentados na Figura 6, assim como os resultados mostrados para as estimações no G1, o nível de amostragem de 0,5 apresentou erros relativos menores que os outros níveis de amostragem estudados. Os outros níveis mostram uma tendência em superestimar os volumes, à medida que se aumenta o tamanho das seções de medição no fuste.

Comparando os resultados das estimativas de $\mathrm{G} 1$ e G2, pode-se notar que ao considerar a influência da base, os resultados das estimativas são mais precisos do que quando não se considera essa influência para todos os níveis de amostragem. Isso pode ser observado nos gráficos de resíduos em que para um mesmo nível de amostragem, há maior valor na superestimação do volume no G2. Pode-se assim afirmar que a base influencia no ajuste, melhorando a estimativa dessa variável. Soares et al. (2010), em seu estudo sobre a influência das seções na estimativa de volume total de fuste de Eucalyptus grandis, concluíram que quando se usa a fórmula de Smalian, seções com escala superior a $2 \mathrm{~m}$ devem ser evitadas, devendo, nesse caso, ser utilizando seções com tamanhos menores na base.

De acordo com a Tabela 4 os valores da estatística $R^{2}$ foram altos para todas as estimativas em todos os níveis de amostragem (superior a 95\%). Porém, as estimativas com influência da base tiveram um resultado mais constante dessa estatística (variação de 96 a 97\%), enquanto o ajuste sem influência da base teve uma maior variação desses valores (variação de 94 a 97\%). Isso pode ser verificado nos gráficos de resíduos sob influência da base, que estimam com maior veracidade os valores das estimativas. Essa mesma regularidade pode ser analisada pela estatística Syx (\%), que apresenta resultados em média mais baixos para o G1 em relação ao G2.

Para a estatística Syx (\%), os valores dos erros nas estimativas do variável volume ficaram intermediários entre os valores apresentados para as variáveis diâmetro e altura. Pode-se notar que, como o volume é a integral da equação de diâmetro, e como essa estatística teve resultados precisos de erros, resultados precisos também podem ser verificados na estimativa dessa variável, que apresentou no G1 erros que não superaram o menor erro apresentado pela variável altura.

Para as estatísticas $V, M D$ e DPD (Tabela 5), os valores das estatísticas para as estimativas nos diferentes níveis de amostragem para o G1 e G2 foram precisos, não ultrapassando o valor da unidade. As 
tendências das estimativas, como observadas na análise gráfica, superestimam os valores das variáveis, à medida que se aumenta o nível de amostragem e diminui o número de seções. Mas, essas tendências considerando cada nível de amostragem foram menores no grupo que leva em consideração a influência da base. Já para a estatística média das diferenças, nas diferentes intensidades de amostragem para ambos os grupos, a precisão dos ajustes foi melhor no G1.

Confrontando os resultados, pode-se notar que as estimativas do G1 foram mais precisas que as estimativas do G2, demonstrando menor superestimação das estimativas de volume. Pode-se também afirmar que a presença de mais medições na base oferece uma melhor estimativa do volume no nível de amostragem considerado.

\section{CONCLUSÕES}

De acordo com os resultados apresentados, pode-se inferir que: As estimativas de diâmetro foram menos tendenciosas e mais precisas, quando as estimativas dos parâmetros utilizados vieram dos ajustes com influência da base.

As estimativas de altura foram mais precisas nos resultados do grupo com influência da base, mostrando que mesmo as funções de afilamento não sendo precisas em estimar valores pequenos dessa variável, esses valores mensurados utilizados no ajuste, ajudam nas estimativas das seções com maior magnitude no fuste.

Para a variável volume, os resultados considerando a influência da base foram mais precisos nos resultados de todos os níveis de amostragem em comparação ao grupo sem influência da base. A influência de medir o maior número de seções na base foi mais precisa nos resultados nas estimativas das três variáveis apresentadas, principalmente para o volume total estimado.

\section{REFERÊNCIAS}

ANGELO, H.; CASTRO, L. H. R.; HOSOKAWA, R. T.; KIRCHNER, F. F.. Análise de componentes principais e função spline para definir a forma do tronco de pinus tropicais. Revista Floresta, v.25, n.12, p.55-67, 1995.

CAMPOS, J. C. C.; LEITE, H. G.. Mensuração florestal: perguntas e respostas. 3.ed. Viçosa: UFV, 2009.

FERREIRA, S. O.. Estudo da forma do fuste de Eucalyptus grandis e Eucalyptus cloeziana. Dissertação (Mestrado em Engenharia Florestal) - Universidade Federal de Lavras, Lavras, 1999.

GOULDING, C. J.. Cubic spline curves and calculation of volume of sectionally measured trees. New Zealand Journal of Forest Science, v.9, n.1, p.89-99, 1979.

HUSCH, B.; BEERS, T. W.; KERSHAW JUNIOR, J. A.. Forest mensuration. 4 ed. Hoboken, New Jersey: John Wiley \& Sons, 2003.

KVALSETH, T. O.. Cautionary note about $R^{2}$. The American Statistican, v.39, n.4, p.279-285, 1985.
MÔRA, R.; SILVA, G. F.; GONÇALVES, F. G.; SOARES, C. P. B.; CHICHORRO, J. F.; CURTO, R. A. F.. Análise de diferentes formas de ajuste de funções de afilamento. Scientia Forestalis, v.42, n.102, p.237-249, 2014.

RATKOWSKY, D. A.. Handbook of nonlinear regression models. New York: Marcel Dekker Inc., 1990.

REGAZZI, A, J.; SILVA, C. H. O.. Testes para verificar a igualdade de parâmetros e a identidade de modelos de regressão não-linear em dados de experimento com delineamento em blocos casualizados. Revista Ceres, v. 57, n.3, p.315-320, 2010.

REGAZZI, A. J.. Teste para verificar a igualdade de parâmetros e a identidade de modelos de regressão nãolinear. Revista Ceres, v.50, n.287, p.9-26, 2003.

SOARES, C. P. B.; PAULA NETO, F.; SOUZA, A. L.. Dendrometria e inventário florestal. Viçosa: UFV, 2006.

SOARES, C. P. B.; SILVA, G. F.; MARTINS, F. B.. Influence of section lenghts on volume determination in Eucalyptus trees. Revista Cerne, v.16, n.2, p.155-162, 2010. 
SOUZA, C. A. M.; SILVA, G. F.; XAVIER, A. C.; MENDONÇA, A. R.; ALMEIDA, A. Q.. Avaliação de modelos de taper não segmentados na estimação da altura e volume comercial de Eucalyptus sp. Ciência Florestal, v.18, n.3, p.387-399, 2008.

A CBPC - Companhia Brasileira de Produção Científica (CNPJ: 11.221.422/0001-03) detém os direitos materiais desta publicação. Os direitos referem-se à publicação do trabalho em qualquer parte do mundo, incluindo os direitos às renovações, expansões e disseminações da contribuição, bem como outros direitos subsidiários. Todos os trabalhos publicados eletronicamente poderão posteriormente ser publicados em coletâneas impressas sob coordenação da Sustenere Publishing, da Companhia Brasileira de Produção Científica e seus parceiros autorizados. Os (as) autores (as) preservam os direitos autorais, mas não têm permissão para a publicação da contribuição em outro meio, impresso ou digital, em português ou em tradução. 